\title{
Predicción de la quiebra en las empresas. Una revisión de literatura*
}

\author{
[Artículos]
}

\section{Martha Teresa Bernate Valbuena** \\ Fabio Enrique Gómez Meneses***}

Recibido: 20 de enero de 2021

Aprobado: 31 de marzo de 2021

Citar como:

Bernate, M. y Gómez, F. (2021). Predicción de la quiebra en las empresas.

Una revisión de literatura. Revista Activos, 19(1).

https://doi.org/10.15332/25005278.6684

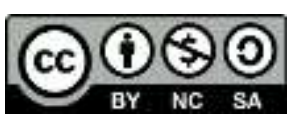

\section{Resumen}

El entorno actual en el que operan las empresas a nivel mundial implica una amplia gama de riesgos que pueden llegar a ocasionar que las compañías se declaren en bancarrota y desaparezcan. Resulta fundamental lograr prever de alguna manera este tipo circunstancias, razón por la cual múltiples autores han abordado este tema con diversos resultados. En este documento se realiza una revisión de la literatura

\footnotetext{
* Este artículo se deriva del trabajo de investigación titulado "La relación entre la calidad de la información contable, la quiebra de las empresas y el desarrollo humano", culminado en el año 2019 en la Universidad de Monterrey, México.

** Doctor en Contabilidad y Finanzas. Profesora de la Escuela de Negocios de la Universidad de Monterrey, México. Correo electrónico: martha.bernate@udem.edu; ORCID:

https://orcid.org/0000-0001-7410-9509

** Doctor en Contabilidad y Finanzas. Profesor de la Escuela de Negocios de la Universidad de Monterrey, México. Correo electrónico: fabio.gomezm@udem.edu; ORCID:

https://orcid.org/0000-0002-7234-6639
}

\section{Revista Activos}

ISSN: 0124-5805 | e-ISSN: 2500-5278 | DOI: https://doi.org/10.15332/25005278

Vol. 19 N.o 1 | enero-junio de 2021 
existente sobre quiebra empresarial durante un periodo de 68 años (1950-2017), espacio de tiempo que supuso un análisis de 4439 trabajos publicados mediante técnicas como el análisis de concurrencia de palabras clave y las redes de citas. Esta amplia revisión permitió identificar dos claras tendencias de investigación al respecto: la primera, relacionada con la estructura financiera de las empresas y la deuda; la segunda, en torno a publicaciones relacionadas con técnicas, métodos y aspectos estadísticos de la predicción de la quiebra empresarial.

Palabras clave: quiebra empresarial, estructura financiera, deuda, capital, bibliometría.

Clasificación JEL: L29, M19.

\section{Predicting bankruptcy in companies. A literature review}

\section{Abstract}

The current environment in which companies operate worldwide involves a wide range of risks that can lead companies to declare bankruptcy and disappear. It is essential to somehow be able to foresee these types of circumstances, which is why many authors have addressed this issue with different results. This paper reviews the existing literature on business bankruptcy over a 68-year period (1950-2017), a time period that involved an analysis of 4,439 papers published using techniques such as keyword concurrency analysis and citation networks. This extensive review allowed the identification of two clear research trends in this regard: the first, related to the financial structure of companies and debt; the second, around publications related to techniques, methods, and statistical aspects of business bankruptcy prediction.

Keywords: business bankruptcy, financial structure, debt, capital, bibliometrics.

JEL classification: L29, M19. 


\section{Introducción}

El éxito o fracaso empresarial es un tema recurrente de investigación desde hace ya varias décadas y, a pesar de que ha sido un tema ampliamente desarrollado, no pierde vigencia debido al significativo impacto que tiene en el desempeño de la economía de un país o de una región. Muchos de los estudios formulados al respecto terminan planteando diversos modelos de predicción cuyo objetivo principal es tratar de identificar una serie de variables que alerten a los administradores de una organización empresarial sobre el posible riesgo de caer en situaciones o escenarios de quiebra empresarial.

En cualquier investigación relacionada con el éxito o fracaso empresarial, resulta muy relevante hacer un barrido, con la mayor profundidad posible, de las diferentes investigaciones existentes al respecto. En el presente trabajo, dicho barrido se presenta con un enfoque distinto a las revisiones llevadas a cabo por autores como Balcaen y Ooghe (2006), Alaka et al. (2017) y Tian et al. (2015).

La revisión de la literatura que se presentará en este documento está basada en el estudio y análisis de datos objetivos, siguiendo un enfoque cienciométrico. Esta revisión fue aplicada para un total de 4439 documentos, sobre los cuales se efectuó un análisis de coocurrencia de palabras clave y un análisis de redes de citas, los cuales fueron el insumo necesario para construir los mapas de conocimiento de la quiebra empresarial.

Básicamente con el desarrollo de este trabajo se buscaba saber, con la mayor confiabilidad posible, cuáles son las temáticas y el alcance de los estudios realizados y publicados sobre el tópico de quiebra empresarial en las últimas 7 décadas, específicamente analizando publicaciones desde el año 1950 y hasta el año 2017. 
Los resultados obtenidos evidenciaron claramente dos corrientes de investigación: la primera, que se enfoca en aspectos como la estructura financiera de la empresa y su deuda, y, la segunda, centrada en las técnicas, métodos y aspectos de tipo estadístico relacionados con la quiebra empresarial. Ahora bien, a partir de estos resultados se hacen una serie de contribuciones que diferencian este trabajo de los aportes realizados por otros autores, por ejemplo, la construcción de los mapas de conocimiento respecto a cómo ha evolucionado la investigación sobre la quiebra empresarial y la identificación de tendencias de investigación emergentes gracias al análisis de palabras clave y el análisis de citas. Todo lo mencionado en los párrafos precedentes se presenta bajo la siguiente estructura. Inicialmente se describe el concepto de 'quiebra empresarial' a partir de los puntos de vista de diversos autores al respecto. Posteriormente, se relaciona el análisis bibliométrico (siguiendo un enfoque cienciométrico) de todas aquellas publicaciones relacionadas con la temática de quiebra empresarial. Allí se indica la metodología, la evolución que han tenido este tipo de publicaciones a lo largo del tiempo y las publicaciones de mayor impacto al respecto. En los siguientes dos apartados, se presenta el análisis de coocurrencia de palabras clave y el análisis de redes de citas, para luego presentar, con base en los anteriores insumos, una revisión general por temática de las publicaciones sobre quiebra y un análisis de las tendencias emergentes al respecto. Por último, se presentan las conclusiones obtenidas en el desarrollo del trabajo.

\section{El concepto de quiebra empresarial}

El concepto de 'quiebra empresarial' puede resultar muy amplio o ambiguo, dependiendo del autor que lo plantee, o si se trata de una definición desde el punto de vista legal. Para Gitman et al. (2015), la quiebra o fracaso empresarial es una circunstancia infortunada que les 
ocurre a las organizaciones empresariales como resultado de una o varias causas que la pueden llevar a desaparecer. Esta situación normalmente ocurre dentro de los primeros años de vida de una compañía, aunque también existen empresas que logran crecer, madurar y quiebran mucho tiempo después.

Lev (1978) plantea que una empresa se puede considerar que fracasa cuando presenta pérdidas continuas o que la rentabilidad financiera de la compañía está por debajo de la que se obtendría en el mercado para una idéntica inversión. Zacharakis et al. (1999) entienden el fracaso empresarial en dos situaciones concretas: cuando el negocio se liquida para evitar nuevas pérdidas y cuando el valor de la empresa está por debajo del costo que implica mantenerla operando.

Otro de los autores que conceptualiza al respecto es Romero (2013), que se refiere específicamente al término de 'fracaso empresarial' cuando se presenta la incapacidad de pagar deudas u obligaciones, la declaración legal de suspensión de pagos o quiebra y la situación patrimonial de una compañía implica un posible fracaso futuro.

Siguiendo esta misma línea, autores como Lizarraga (1997) vinculan los términos de 'quiebra' y 'fracaso empresarial' con la insolvencia; es decir, una compañía quebrará o fracasará cuando sea insolvente. No obstante, aquí habría que tener en cuenta si se está hablando de insolvencia aguda o técnica, insolvencia crónica o insolvencia terminal o definitiva. La primera se refiere a la falta de liquidez en el corto plazo para tender pasivos vencidos, la segunda hace alusión a la falta de liquidez para el pago de pasivos que involucran dos o más ejercicios económicos y la tercera se refiere a la falta de capacidad para financiar cambios perdurables en el balance general de la compañía. 
Para autores como Ibarra (2002) y Manzaneque et al. (2010), la quiebra o fracaso empresarial involucra un proceso de crisis que se desarrolla mediante una serie de etapas: el fracaso económico, donde los ingresos no cubren los costos totales; el fracaso financiero, cuando la empresa no puede pagar a sus deudas por falta de liquidez a medida que estas están venciendo, y, finalmente, el fracaso legal, etapa que implica los procesos concursales y judiciales, y la desaparición legal de la organización.

Desde un punto de vista legal y según lo plantean Gitman et al. (2015), y García-Gallego y Mures-Quintana (2013), la quiebra ocurre cuando una empresa no puede pagar sus deudas o cuando sus pasivos exceden el valor de mercado de sus activos. Con esto, la organización podría ser legalmente declarada en bancarrota, claro está, dependiendo de la legislación vigente y de los procedimientos legales que haya que llevar a cabo en cada país.

Teniendo en cuenta todos los conceptos anteriormente indicados y de acuerdo con el contexto de este trabajo, para la identificación de los diferentes artículos y documentos, se utilizó la expresión en inglés bankruptcy y sus diferentes sinónimos, como por ejemplo "failure", "business failure", "insolvency" y "default", tal cual es explicado en el siguiente apartado de este documento.

\section{Análisis bibliométrico con enfoque cienciométrico de las publicaciones relacionadas con la temática de quiebra empresarial}

La revisión de la literatura que se hace en esta investigación sigue un enfoque cienciométrico, es decir, se basa en el análisis de datos objetivos a partir de la coocurrencia de palabras clave y de redes de citas; con dicha información, se logran obtener los mapas de conocimiento del avance en la investigación en lo que concierne a la temática de quiebra empresarial. La 
explicación y detalle de un análisis de coocurrencia de palabras clave y un análisis de redes de citas se presenta en los apartados 4 y 5 del documento. Para llevar a cabo la búsqueda de publicaciones relacionadas con el tema de quiebra empresarial, se consideraron inicialmente las tres bases de datos bibliométricas más importantes en la actualidad: Web of Science, Scopus y Google Scholar. En lo que concierne a Scopus, esta fue descartada debido a que se enfoca a artículos publicados desde el año 1995, de modo que no cubría la serie de tiempo elegida en esta investigación. Respecto a Google Scholar, tampoco se tuvo en cuenta debido a la evidente generalidad y menor precisión de sus resultados. Teniendo en cuenta lo anterior, la base de datos seleccionada fue Web of Science.

La búsqueda de las publicaciones se ejecutó teniendo en cuenta parámetros como el título, el resumen y las palabras clave, utilizando la expresión en inglés "bankruptcy" y sus diferentes sinónimos. Se utilizó la siguiente cadena de búsqueda para estos tres parámetros:

TS = (bankruptcy) OR TS = ("Business failure") OR TS = ("Business failures") OR TS = ("financial distress") OR TS = ("bankruptcy prediction”) OR TS = ("predictors of failure")

Esta cadena de búsqueda generó un total de 8435 casos desde el año 1950 hasta el año 2017. Esta cifra fue depurada teniendo en cuenta documentos en los cuales no se pudiera evidenciar claramente el título y resumen, lo cual generó que el número de casos se redujera a 3173. Finalmente, se excluyeron aquellos registros que tuvieran un bajo impacto de acuerdo con el número de citas en Google y en Web of Science, con lo cual se obtuvo una muestra final de 25 casos. En la figura 1 se presenta de forma más específica cómo se llevó a cabo el proceso de selección de datos. 
Figura 1. Proceso de selección de los datos de la muestra

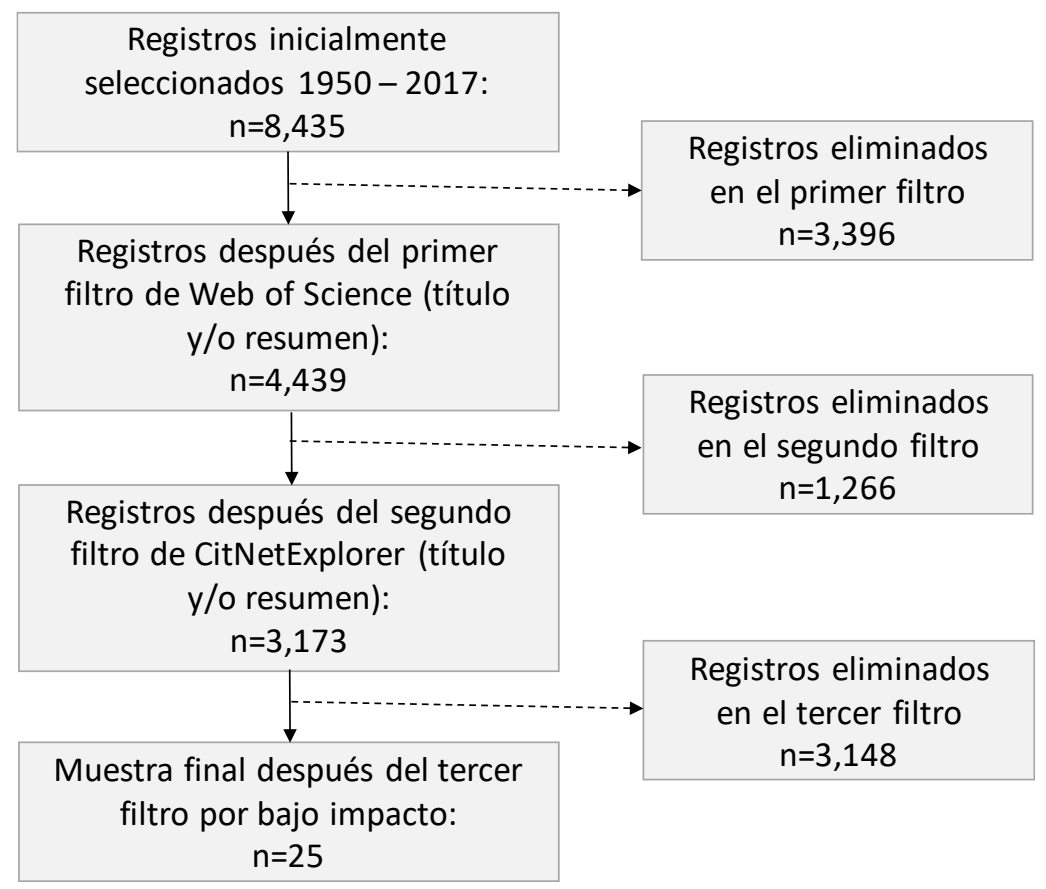

Fuente: elaboración propia.

Los resultados derivados de esta búsqueda permiten establecer un panorama general respecto a las publicaciones sobre la temática de quiebra empresarial. Durante un periodo de casi dos décadas, que se extiende desde 1950 hasta 1966, las investigaciones o trabajos publicados al respecto son muy escasos. A pesar de lo anterior, los pocos documentos publicados en esos años significaron el punto de partida para que la comunidad científica se interesará al respecto, trabajos como los de Beaver (1966) y Altman (1968) dieron este primer paso que dio significativos frutos en años posteriores.

A partir de la década de 1970 y hasta el año de 1991, los trabajos sobre quiebra empresarial comenzaron a aumentar, aunque fuera muy lento. Esta tendencia cambia poco a poco a partir del año 1992 y hasta el año 2004, donde claramente se evidencia un aumento en este tipo de publicaciones, aunque no es significativo. A partir del año 2005, se 
comienza a observar un aumento muy significativo en el número de publicaciones sobre quiebra empresarial, ya que se pasa de una cifra aproximada de 70 u 80 artículos publicados por año, durante los primeros años de la década del 2000, a más de 400 artículos publicados en el año 2017.

En el párrafo anterior se pueden apreciar dos puntos de inflexión: el primero, en el periodo comprendido entre 1992 y 2004 y, el segundo, a partir del año 2005. La posible explicación a este incremento en el número de publicaciones para el primer periodo está dada por la burbuja de las punto-com y su posterior descalabro; en lo que concierne al segundo periodo, este se justifica seguramente por la crisis económica mundial que empezó en Estados Unidos en el año 2008 y se extendió por varios años a través de todo el planeta. En la figura 2 se pueden apreciar de una forma mucho más clara estos dos puntos de inflexión y, en general, cómo ha sido la evolución de las publicaciones sobre quiebra empresarial a nivel mundial. 
Figura 2. Publicaciones sobre quiebra empresarial 1967-2017

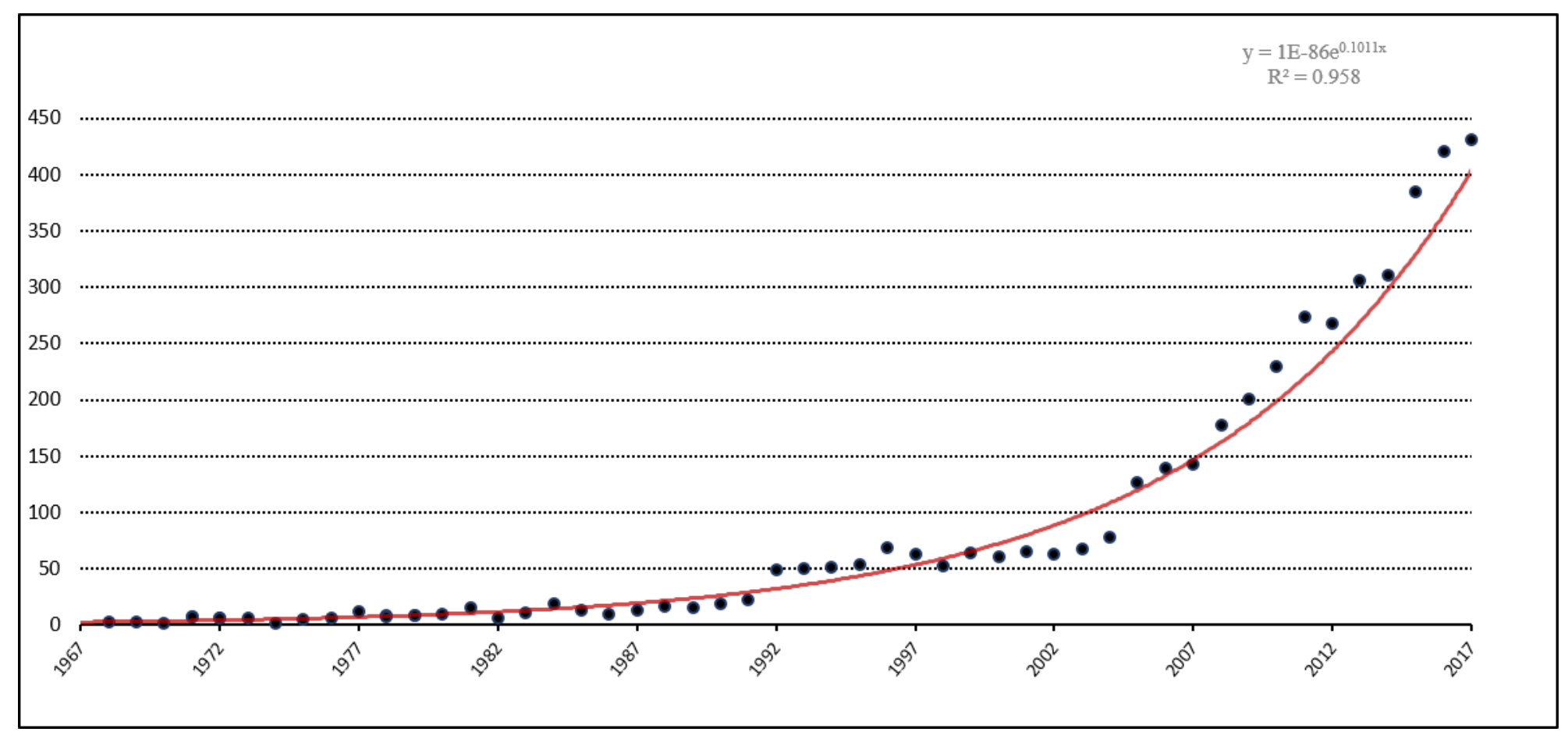

Fuente: elaboración propia, a partir de Web of Science (2018). 
Ahora bien, de todas estas publicaciones obtenidas en la búsqueda, se seleccionaron las 25 más representativas o de mayor impacto, teniendo como criterio de selección el número de citas obtenidas por cada documento. Todos estos trabajos fueron publicados en prestigiosas revistas como Journal of Finance, Journal of Accounting Research o American Economic Review. En la tabla 1 se presenta la información bibliométrica de los 25 trabajos a los cuales se hace alusión en su totalidad.

Tabla 1. Artículos publicados de mayor impacto 1950-2017

\begin{tabular}{|c|c|c|c|c|}
\hline Artículo & Revista & $\begin{array}{l}\text { Citas de } \\
\text { Scholar } \\
\text { Google / } \\
\text { (ranking) }\end{array}$ & $\begin{array}{l}\text { Citas de } \\
\text { Web of } \\
\text { Science / } \\
\text { (ranking) }\end{array}$ & $\begin{array}{c}\text { Citas de } \\
\text { investigadores } \\
\text { de quiebra / } \\
\text { (ranking) }\end{array}$ \\
\hline Altman (1968) & Journal of Finance & 15119 / (4) & $2893 /(6)$ & $916 /(1)$ \\
\hline Altman (1984) & Journal of Finance & $1556 /(16)$ & $271 /(25)$ & $112 /(25)$ \\
\hline Andrade y Kaplan (1998) & Journal of Finance & 1392 / (18) & $307 /(23)$ & $119 /(21)$ \\
\hline Bharath y Shumway (2008) & $\begin{array}{c}\text { The Review of Financial } \\
\text { Studies }\end{array}$ & $1176 /(22)$ & $339 /(18)$ & $131 /(16)$ \\
\hline Beaver (1966) & $\begin{array}{c}\text { Journal of Acounting } \\
\text { Research }\end{array}$ & $6378 /(7)$ & $881 /(8)$ & $361 /(4)$ \\
\hline Black y Scholes (1973) & Journal of Political Economy & $35860 /(1)$ & $8191 /(1)$ & 187 / (9) \\
\hline Campbell et al. (2008) & Journal of Finance & $1576 /(15)$ & $365 /(16)$ & $126 /(17)$ \\
\hline Gilson et al. (1990) & $\begin{array}{c}\text { Journal of Financial } \\
\text { Economics }\end{array}$ & 1284 / (19) & $322 /(21)$ & $138 /(14)$ \\
\hline Gruber y Warner (1977) & The Journal of Finance & 1194 / (21) & $368 /(15)$ & $123 /(19)$ \\
\hline Hillegeist et al. (2004) & $\begin{array}{c}\text { Review of Accounting } \\
\text { Studies }\end{array}$ & $1206 /(20)$ & 337 / (19) & $177 /(10)$ \\
\hline Jensen (1986) & American Economic Review & $26237 /(2)$ & $5496 /(2)$ & 194 / (8) \\
\hline Leland (1994) & The Journal of Finance & 2879 / (9) & $691 /(10)$ & $177 /(10)$ \\
\hline Leland y Toft (1996) & The Journal of Finance & $2358 /(12)$ & $513 /(13)$ & $132 /(15)$ \\
\hline Merton (1974) & The Journal of Finance & $12138 /(6)$ & $3456 /(4)$ & $370 /(3)$ \\
\hline Min y Lee (2005) & $\begin{array}{l}\text { Expert Systems with } \\
\text { Applications }\end{array}$ & $711 /(24)$ & $324 /(20)$ & $126 /(17)$ \\
\hline Modigliani y Miller (1958) & $\begin{array}{c}\text { The American Economic } \\
\text { Review }\end{array}$ & $22616 /(3)$ & $3715 /(3)$ & $160 /(12)$ \\
\hline Myers (1977) & $\begin{array}{c}\text { Journal of Finance } \\
\text { Economics }\end{array}$ & 14669 / (5) & $3057 /(5)$ & $234 /(7)$ \\
\hline
\end{tabular}

Revista Activos

ISSN: 0124-5805 | e-ISSN: 2500-5278 | DOI: https://doi.org/10.15332/25005278

Vol. 19 N.o 1 | enero-junio de 2021 


\begin{tabular}{|c|c|c|c|c|}
\hline Artículo & Revista & $\begin{array}{l}\text { Citas de } \\
\text { Scholar } \\
\text { Google / } \\
\text { (ranking) }\end{array}$ & $\begin{array}{l}\text { Citas de } \\
\text { Web of } \\
\text { Science / } \\
\text { (ranking) }\end{array}$ & $\begin{array}{c}\text { Citas de } \\
\text { investigadores } \\
\text { de quiebra / } \\
\text { (ranking) }\end{array}$ \\
\hline Ohlson (1980) & $\begin{array}{c}\text { Journal of Accounting } \\
\text { Research }\end{array}$ & $6030 /(8)$ & $1288 /(7)$ & $561 /(2)$ \\
\hline Opler y Titman (1994) & The Journal of Finance & $1537 /(17)$ & 344 / (17) & $116 /(24)$ \\
\hline Shleifer y Vishny (1992) & Journal of Finance & $2644 /(11)$ & $651 /(11)$ & $117 /(23)$ \\
\hline Shin et al. (2005) & $\begin{array}{l}\text { Expert Systems with } \\
\text { Applications }\end{array}$ & 694 / (25) & $316 /(22)$ & $120 /(20)$ \\
\hline Shumway (2001) & The Journal of Business & $2348 /(13)$ & $650 /(12)$ & $282 /(6)$ \\
\hline Vassalou y Xing (2004) & The Journal of Finance & 1897 / (14) & $458 /(14)$ & $139 /(13)$ \\
\hline Wilson y Sharda (1994) & Decision Support Systems & $810 /(23)$ & $280 /(24)$ & $119 /(21)$ \\
\hline Zmijewski (1984) & $\begin{array}{c}\text { Journal of Accounting } \\
\text { Research }\end{array}$ & $2741 /(10)$ & 695 / (9) & $288 /(5)$ \\
\hline
\end{tabular}

Fuente: elaboración propia, con base en Web of Science y Google Scholar (2018).

Lógicamente no todos los trabajos presentados en este listado hablan exclusivamente del tema de quiebra empresarial. Algunos de ellos abordan una serie de temáticas conexas que resultan absolutamente indispensables para entender todo lo relacionado con la bancarrota en las organizaciones empresariales.

\section{Análisis de coocurrencia de palabras clave}

Para la ejecución del análisis de coocurrencia se siguen los criterios indicados por Radhakrishnan et al. (2017), es decir, se extraen las palabras clave de cada artículo; cada una de ellas se representará por un nodo y un enlace indicará la coincidencia de cada par de palabras. El peso de dicho enlace estará dado por la cantidad de veces que un par de palabras coocurre en varios artículos.

Siguiendo a Van Eck y Waltman (2017), las redes que se originan a partir de este análisis se utilizaron en la construcción de los mapas de conocimiento, mediante el uso del software VOSwiever (versión 1.6.16. 
Específicamente se formuló un mapa del conocimiento global y tres mapas para tres periodos secuenciales. Los tres periodos a los que se hace alusión son:

- Periodo I: de 1967 a 1991

- Periodo II: de 1992 a 2004

- Periodo III: de 2005 a 2017

Es importante mencionar que no se tuvo en cuenta la etapa comprendida entre los años 1950 y 1966 debido a la mínima producción bibliográfica existente respecto a quiebra empresarial. También, durante el primer periodo indicado, las publicaciones no solían incluir las palabras clave; por lo tanto, para solventar esta limitación, se tomaron las palabras contenidas en los títulos de los documentos.

En lo que concierne al primer periodo, en la figura 3 se pueden observar pocos tópicos de interés en pequeñas agrupaciones. Una primera agrupación (izquierda de la figura) corresponde a la temática de ley de quiebra y a la evaluación económica de los procesos de quiebra. De otra parte, las agrupaciones de la derecha de la figura se refieren a la evaluación de la capacidad de los indicadores financieros para la predicción de quiebra; el fracaso empresarial y la quiebra corporativa.

Este análisis permite determinar que, durante el primer periodo objeto de estudio, el foco de investigación se centró en la dificultad financiera en general, es decir, se abordaron aspectos como los costos de quiebra, la quiebra personal y la quiebra corporativa (Myers, 1977; Jensen y Meckling, 1976; Gruber y Warner, 1977; Altman, 1984; Shiers y Williamson, 1987; Ang y Chua, 1981; Rose y Giroux, 1984).

El camino recorrido en este primer periodo empezó con investigaciones sobre indicadores que influyen en el fracaso empresarial (Simpson y Anderson, 1957), para luego pasar por el modelo de análisis univariante de 
Beaver (1966) y, finalmente, llegar al concepto de 'quiebra empresarial' junto con su predicción, liderado por autores como Altman (1968), que aportaron el empleo de técnicas de análisis discriminante multivariante que valoraban la calidad de una serie de indicadores económicosfinancieros de forma conjunta (figura 3).

Para el segundo periodo se pueden observar, con una mayor claridad, dos tendencias o enfoques de investigación (figura 4). El primero de ellos, en la izquierda del gráfico, se refiere al estudio de la empresa y sus dificultades financieras, donde se puede hacer alusión a trabajos como los de Gilson (1990) y Gruber y Warner (1977). Estas investigaciones abordaron temáticas relacionadas como la reestructuración de la quiebra, el costo de la quiebra y la incapacidad de la empresa para obtener créditos o emitir valores. En cuanto al segundo enfoque, en la derecha del gráfico, los trabajos desarrollados exploran los modelos de predicción de quiebra basados en el análisis discriminante multivariante o en las redes neuronales.

Finalmente, en lo que concierne al último periodo, los dos enfoques de investigación comentados en el periodo anterior se acentúan profundamente en esta etapa. De esta manera, se observan estas dos tendencias, pero con muchas más investigaciones, que, para el caso de los estudios sobre empresa y sus dificultades financieras, explora otros componentes adicionales de investigación como, por ejemplo, las combinaciones entre capital y deuda elegidas por los gerentes, y el endeudamiento y su efecto en el valor de las acciones de una compañía. Si se analiza el segundo grupo, se profundiza en la predicción de quiebra mediante redes neuronales (Coats y Fant, 1993) y se incluyen modelos de predicción de quiebra basados en algoritmos genéticos y en máquinas de vectores de soporte (Min y Lee, 2005) (figura 5). 
Figura 3. Mapa de conocimiento en periodo I: 1967-1991

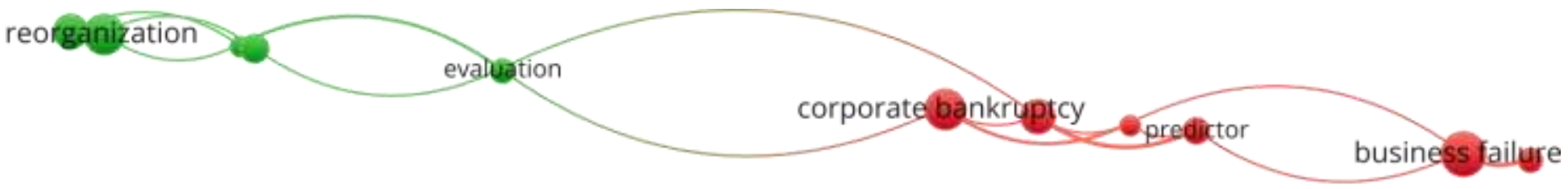

\& vosviewer

Fuente: elaboración propia en VOSviewer. 
Figura 4. Mapa de conocimiento durante el periodo II: 1992-2004

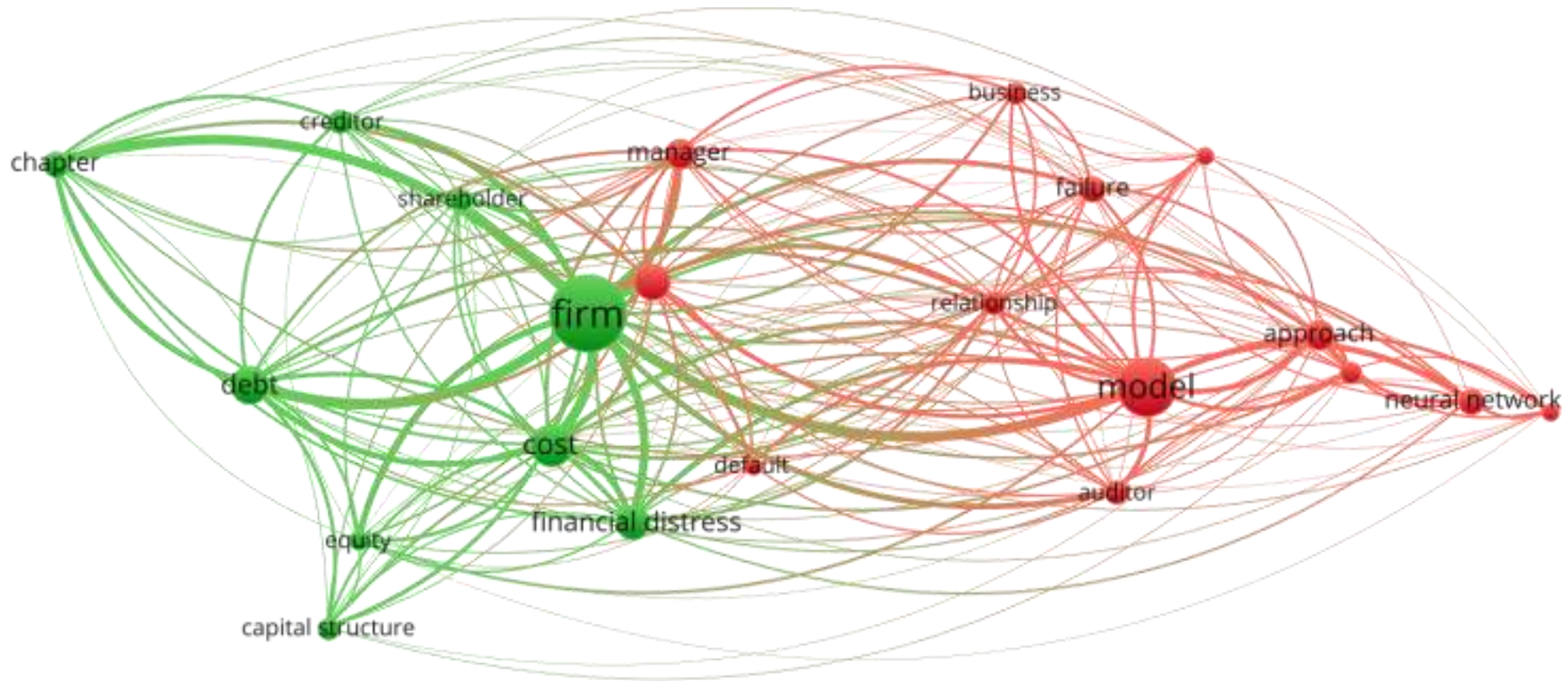

\& VOSviewer

Fuente: elaboración propia en VoSviewer. 
Figura 5. Mapa de conocimiento durante el periodo III: 2005-2017

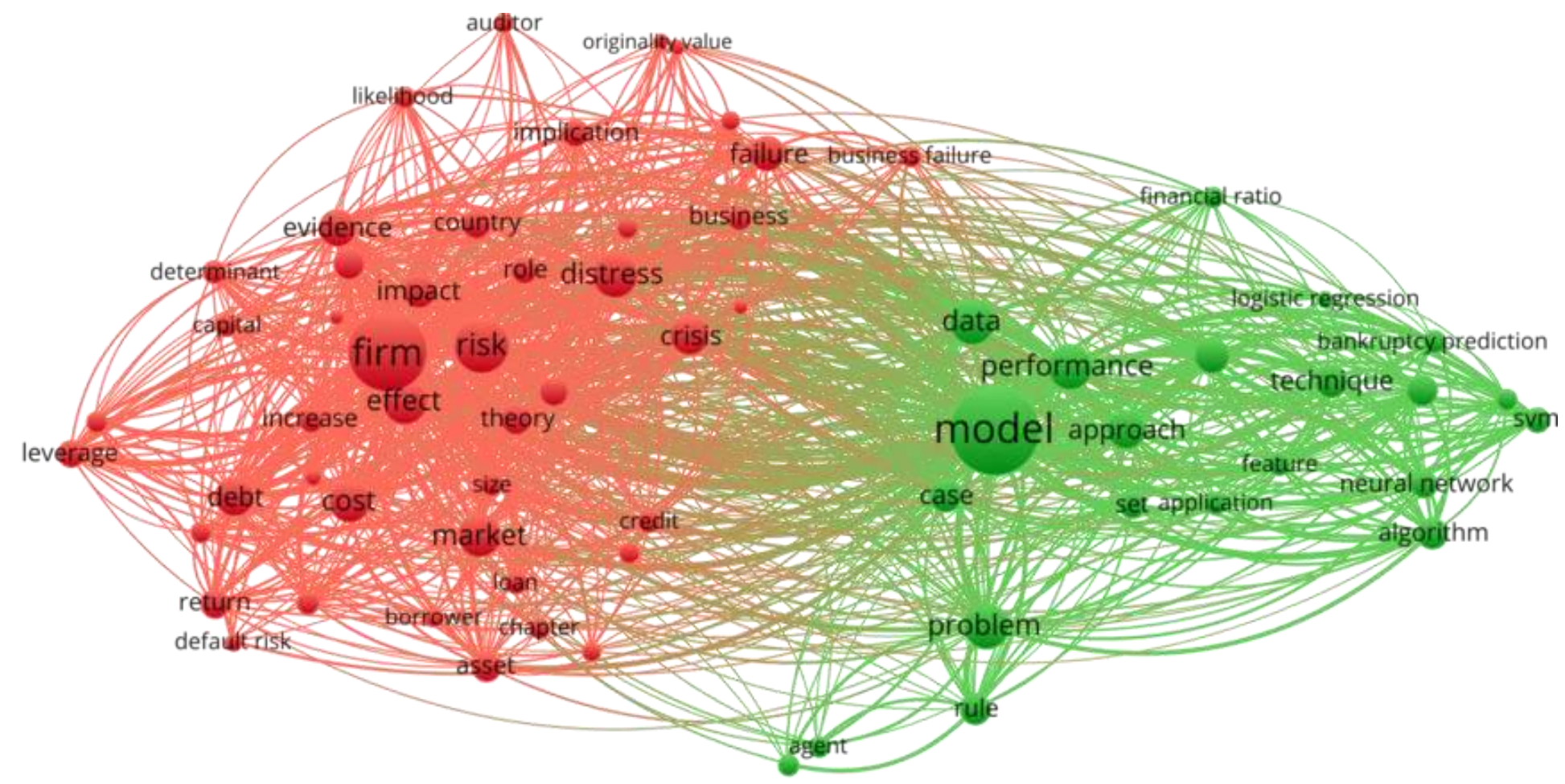

\& VOSviewer

Fuente: elaboración propia en VoSviewer.

ISSN: 0124-5805 | e-ISSN: 2500-5278 | DOI: https://doi.org/10.15332/25005278

Vol. 19 N. ${ }^{\circ} 1$ | enero-junio de 2021 


\section{Análisis de redes de citas}

El análisis de redes de citas básicamente permite observar la influencia de las publicaciones de los autores más citados a lo largo de un periodo de tiempo sobre un tema específico; para el caso de esta investigación, la quiebra empresarial. Este análisis fue realizado mediante el software CitNet Explorer (Centre for Science and Technology Studies, 2014) y dio como resultado el mapa de conocimiento que se presenta en la figura 6 .

Figura 6. Análisis de redes de citación sobre el tema de quiebra empresarial

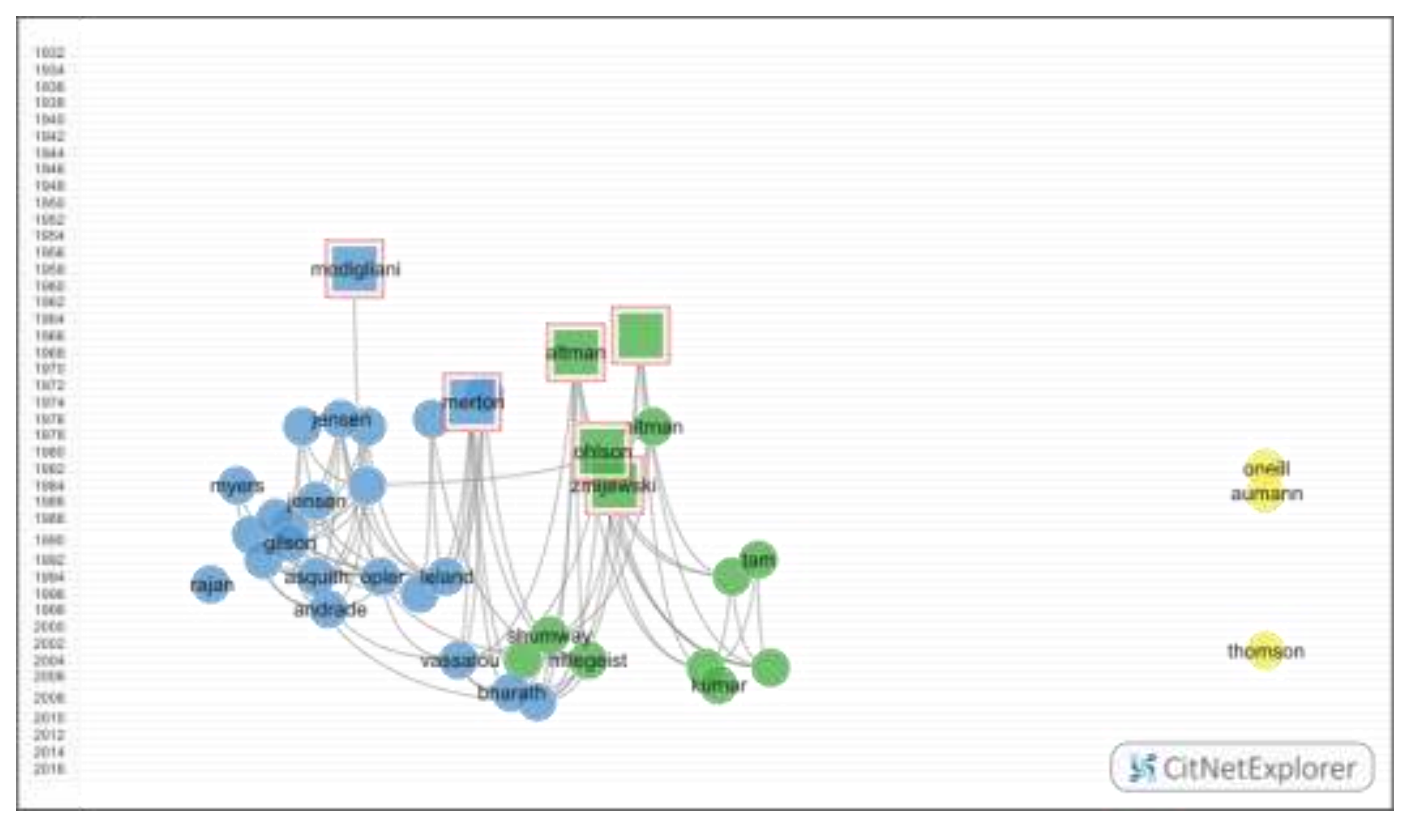

Fuente: elaboración propia con base en datos de Web of Science (2018).

En el mapa anteriormente presentado se confirma lo expuesto en el análisis de palabras clave, es decir, la evidente separación en dos temáticas de estudio: por una parte, los trabajos relacionados con aspectos empresariales como la estructura de capital, la deuda, los costos y la quiebra y, por otro lado, las investigaciones relacionadas con técnicas, métodos y aspectos de tipo estadístico para la creación de modelos de predicción. 


\section{Revisión de las publicaciones sobre quiebra}

Una vez realizado el análisis de palabras clave y el análisis de redes de citas, se identifica una serie de temáticas generales que se han desarrollado a lo largo del tiempo en las publicaciones relacionadas con la quiebra empresarial. Específicamente se puede hablar de tres grandes tópicos:

- Teoría económica

- Causas del fracaso empresarial

- Predicción de la quiebra

\subsection{Teoría económica}

Dentro de esta primera categoría se abordan temas como la teoría de la estructura de capital, la teoría de la valoración del riesgo de crédito y la teoría del costo de la quiebra. Respecto al primero de estos temas, resulta imprescindible mencionar los trabajos de Modigliani y Miller (1958; 1965), estudios en cuales se indicó que el valor de una empresa no se ve afectada por la forma como la compañía se financia. Esta premisa inicial fue complementada, incorporando el elemento de los impuestos, los cuales pueden llegar a ocasionar una ventaja fiscal que incrementa el valor de la empresa al ser está financiada con deuda. Otros trabajos adicionales que complementan lo planteado por Modigliani y Miller son las investigaciones de Jensen y Meckling (1976), Altman (1984) y Leland (1994). Básicamente, estos autores critican el modelo inicial, planteando una serie de cuestionamientos por no considerar otros aspectos como los costes de quiebra y los subsidios tributarios.

Con respecto al segundo de los temas desarrollados "la teoría de la valoración del riesgo de crédito", se pueden mencionar los trabajos de Black y Sholes (1973) y Merton (1974), que dieron origen a la formula Black-Shocles-Merton (BSM), la cual ha sido significativa en la forma 
como los agentes valoran y cubren las opciones. Estudios posteriores confirman la utilidad del modelo BSM sobre las medidas tradicionales basadas en la información contable; dentro de estos trabajos se encuentran los de Hillegeist et al. (2004) y Campbell et al. (2008).

En cuanto al último de los temas desarrollados, la teoría de los costos de quiebra, se pueden citar autores como Leland (1994), Jensen y Meckling (1976), Altman (1984) y Myers y Majluf (1984), quienes indican que en los costos de la dificultad financiera se incluyen los costos de quiebra y los problemas de agencia asociados con la deuda en riesgo. En opinión de Altman (1984), dichos costos son muy relevantes, pues significan entre un $11 \%$ y un $17 \%$ del valor de la empresa, o entre un $10 \%$ y un $17 \%$ de los ingresos operativos, según lo planteado por Andrade y Kaplan (1998).

\subsection{Causas del fracaso empresarial}

Las causas del fracaso empresarial son múltiples. Para Campbell et al. (2008), las empresas que más fácilmente pueden quebrar son aquellas cuyas acciones tienen precios bajos, con una menor capitalización bursátil, con retornos de acciones pasadas más inestables, un menor respaldo de efectivo, un mayor apalancamiento y una menor rentabilidad.

Dentro de la amplia literatura al respecto, se identifican dos grandes focos de investigación relacionados con las causas del fracaso empresarial, el sector y tamaño de la empresa, así como las habilidades y cualidades de la dirección. Para el caso del primer foco de investigación, se pueden mencionar los trabajos de Ooghe y De Prijcker (2008), Bunn y Redwood (2003), Zmijewski (1984), Altman y Hotchkiss (2010) y Situm (2014). La mayoría de estos trabajos establece una relación significativa entre tamaño y probabilidad de quiebra, aunque dependiendo del autor los resultados pueden ser diversos. En estudios como los de Situm (2014), son las empresas pequeñas las que mayor probabilidad tienen de fracasar, 
mientras que, en investigaciones como las de Altman y Hotchkiss (2010), son las grandes empresas las que tienen una mayor probabilidad de fracaso.

Respecto al segundo foco de investigación, las habilidades y cualidades que puedan tener los directores o gerentes de una compañía resultan ser la principal causa del fracaso empresarial, según lo planteado por Ooghe y De Prijcker (2008). Estos autores clasifican a las empresas según el comportamiento de sus directores y dueños (tabla 2).

Tabla 2. Clasificación de las empresas según el comportamiento de sus directores

\begin{tabular}{|c|c|c|}
\hline Tipo de empresa & Características & Perfil financiero \\
\hline $\begin{array}{l}\text { Nuevas empresas sin } \\
\text { éxito }\end{array}$ & $\begin{array}{l}\text { Generalmente, una empresa nueva } \\
\text { tiene mayor riesgo de fracasar; por } \\
\text { tanto, estas deben iniciar con capital } \\
\text { significativo. No obstante, algunas } \\
\text { veces, aunque posean suficiente } \\
\text { capital para iniciar, si no reciben apoyo } \\
\text { de los bancos, tienen escasas } \\
\text { posibilidades de sobrevivir. }\end{array}$ & $\begin{array}{l}\text { Lento deterioro en solvencia y } \\
\text { liquidez posterior a los problemas } \\
\text { de rentabilidad. }\end{array}$ \\
\hline $\begin{array}{l}\text { Crecimiento con base en } \\
\text { el riesgo }\end{array}$ & $\begin{array}{l}\text { Al conservar una postura ambiciosa y } \\
\text { optimista puede incrementar su } \\
\text { endeudamiento. Al proponer nuevas } \\
\text { inversiones o expansiones puede } \\
\text { lograr mantener la rentabilidad, } \\
\text { aunque su solvencia y liquidez serán } \\
\text { frágiles. }\end{array}$ & $\begin{array}{l}\text { Lento crecimiento en la } \\
\text { rentabilidad acompañado de } \\
\text { fragilidad en la solvencia y la } \\
\text { liquidez. }\end{array}$ \\
\hline $\begin{array}{l}\text { Crecimiento } \\
\text { extraordinario }\end{array}$ & $\begin{array}{l}\text { La dirección de la empresa toma } \\
\text { riesgos desmesurados e ignora la } \\
\text { estructura administrativa y operativa } \\
\text { de la compañía. Exagerado aumento } \\
\text { de los gastos y una disminución de la } \\
\text { rentabilidad, aunque la escasa } \\
\text { rentabilidad es atribuida a cuestiones } \\
\text { temporales y del ambiente externo. }\end{array}$ & $\begin{array}{l}\text { Enorme rentabilidad que deriva en } \\
\text { falta de liquidez y solvencia. }\end{array}$ \\
\hline
\end{tabular}

Fuente: elaboración propia, con base en Ooghe y De Prijcker (2008). 


\subsection{Predicción de la quiebra}

En este tercer y último gran tópico, los investigadores desarrollan trabajos relacionados con la predicción de la quiebra. Dentro de los primeros trabajos al respecto, se pueden mencionar los de Smith y Winakor (1930), Smith (1935), FitzPatrick (1932) y Hickman (1958). Después de este primer periodo aparecen los trabajos de Beaver (1966) y Altman (1968), quienes posicionan sus trabajos como los de mayor impacto en el ámbito de la evaluación y predicción del fracaso empresarial, mediante la utilización de la información contenida en la contabilidad de las empresas y la información suministrada por las ratios financieras. Finalmente, en un último periodo se plantean diversos modelos de predicción de quiebra, que, según Adnan Azis y Dar (2006), pueden ser clasificados en tres categorías:

- Modelos teóricos

- Modelos empíricos

- Modelos basados en inteligencia artificial

En las tablas 3, 4 y 5 se muestran algunos ejemplos de modelos de predicción de quiebra en cada una de estas tres categorías.

Tabla 3. Modelos teóricos de predicción de quiebra

\begin{tabular}{l|l}
\multicolumn{1}{c|}{ Teoría } & \multicolumn{1}{c}{ Características } \\
\hline $\begin{array}{l}\text { Medidas de } \\
\text { descomposición del } \\
\text { balance - Balance } \\
\text { sheet decomposition } \\
\text { measures }\end{array}$ & $\begin{array}{l}\text { Analiza la estructura de los estados financieros, para identificar la dificultad } \\
\text { financiera. Si se presentan cambios significativos en la estructura de activos y } \\
\text { pasivos, hay probabilidad de que la empresa sea incapaz de mantener el estado } \\
\text { de equilibrio; si dichos cambios a futuro se vuelven incontrolables, se puede } \\
\text { vislumbrar que la empresa presenta dificultades financieras. }\end{array}$ \\
\hline $\begin{array}{l}\text { Administración de } \\
\text { efectivo - Cash } \\
\text { Management Theory }\end{array}$ & $\begin{array}{l}\text { Analiza la administración del efectivo. Si se presenta un desequilibrio de } \\
\text { entradas y salidas de efectivo y perdura en el tiempo, podría causar dificultades } \\
\text { financieras y por tanto la quiebra empresarial. }\end{array}$
\end{tabular}




\begin{tabular}{|c|c|}
\hline Teoría & Características \\
\hline \multirow{3}{*}{$\begin{array}{l}\text { Teorías del Riesgo de } \\
\text { crédito - Credit risk } \\
\text { theories } \\
\text { Analizan el riesgo de } \\
\text { crédito en base a los } \\
\text { acuerdos de Basilea I } \\
\text { y II. Aplica } \\
\text { principalmente a } \\
\text { empresas financieras. } \\
\text { El riesgo tratado en } \\
\text { estas teorías es el } \\
\text { riesgo de cualquier } \\
\text { prestatario que } \\
\text { incumpla. }\end{array}$} & $\begin{array}{l}\text { Enfoque de migración de crédito propuesto por JP Morgan con CreditMetrics, y } \\
\text { Moody's KMV. Basado en la teoría del precio de las opciones, donde el } \\
\text { incumplimiento está endógenamente relacionado con la estructura de capital y } \\
\text { la empresa puede incumplir sus obligaciones si el valor de sus activos cae por } \\
\text { debajo de un nivel crítico (determinado por el modelo de riesgo crediticio). } \\
\text { Tiene como fundamento el modelo de valor de activos originalmente propuesto } \\
\text { por Merton (1974). }\end{array}$ \\
\hline & $\begin{array}{l}\text { Enfoque actuarial propuesto por Credit Suisse Financial Products (CSFP) con } \\
\text { CreditRisk. Centrado en el incumplimiento de los bonos o préstamos } \\
\text { individuales. }\end{array}$ \\
\hline & $\begin{array}{l}\text { CreditPortfolioView. Modelo de periodo múltiple de tiempo donde las } \\
\text { probabilidades de incumplimiento son condicionales a las macrovariables como } \\
\text { el desempleo, la tasa de crecimiento en la economía y el nivel de las tasas de } \\
\text { interés, que en gran medida impulsan el ciclo de crédito en la economía. }\end{array}$ \\
\hline
\end{tabular}

Fuente: elaboración propia en base a Crouhy et al. (2000) y Adnan Aziz y

Dar (2006).

Tabla 4. Modelos empíricos de predicción de quiebra

\begin{tabular}{|l|l|}
\hline \multicolumn{1}{|c|}{ Modelo } & \multicolumn{1}{c}{ Características } \\
\hline Univariante & $\begin{array}{l}\text { Centrado en el análisis de ratios financieros. Si los ratios presentan diferencias } \\
\text { significativas entre las empresas que fracasan y las que no, entonces pueden usarse } \\
\text { como variables predictivas. Un representante de este modelo es Beaver (1966). }\end{array}$ \\
\hline $\begin{array}{l}\text { Enálisis } \\
\text { discriminante combinación lineal (una puntuación de quiebra) de determinadas variables } \\
\text { múltiple }\end{array}$ & $\begin{array}{l}\text { discriminatorias que se utiliza para clasificar las empresas en grupos de quiebra y no } \\
\text { quiebra en función de sus características especiales. Altman (1968) lo utilizó por primera } \\
\text { vez para desarrollar modelo de predicción de bancarrota, popularmente conocido como } \\
\text { modelo Z, que mejora el trabajo univariante de Beaver (1966). Este modelo se } \\
\text { popularizó posteriormente en la literatura contable y financiera. }\end{array}$ \\
\hline Probit & $\begin{array}{l}\text { Por medio de una variable dicotómica, que es una función lineal de un vector de } \\
\text { variables explicativas, indica la probabilidad de fracaso o éxito. Zmijewski (1984) fue } \\
\text { pionero en la utilización de esta técnica. }\end{array}$ \\
\hline $\begin{array}{l}\text { Utiliza la misma metodología que la probabilidad lineal, aunque el logaritmo es un } \\
\text { logaritmo de probabilidad de que ocurra el evento de fracaso o no fracaso. En la }\end{array}$ \\
$\begin{array}{l}\text { solicitud de quiebra, una probabilidad de 0.5 implica una igual posibilidad de que la } \\
\text { empresa quiebre o no quiebre. Por lo tanto, donde 0 indica quiebra, cuanto más cerca } \\
\text { está el estimado a 1, menos posibilidades hay de que la empresa quiebre. Ohlson (1980) } \\
\text { fue pionero en el análisis logit utilizando variables contables para estimar la probabilidad } \\
\text { de quiebra. Su medida al igual que el Z-score de Altman se ha utilizado, por ejemplo, por } \\
\text { Dichev (1998), Griffin y Lemmon (2002), y Ferguson y Shockley (2003), Campbell et al. } \\
\text { (2008) y Shumway (2001). }\end{array}$ \\
\hline Logit
\end{tabular}

Fuente: elaboración propia en base a Alaka et al. (2017) y Adnan Aziz y

Dar (2006).

Revista Activos

ISSN: 0124-5805 | e-ISSN: 2500-5278 | DOI: https://doi.org/10.15332/25005278

Vol. 19 N. ${ }^{\circ} 1$ | enero-junio de 2021 
Tabla 5. Modelos de inteligencia artificial para predicción de quiebra

\begin{tabular}{|c|c|}
\hline Modelo & Características \\
\hline $\begin{array}{l}\text { Arboles de decisión } \\
\text { o decision trees }\end{array}$ & $\begin{array}{l}\text { Es una forma de aprendizaje supervisado en el que un programa aprende } \\
\text { generalizando a partir de ejemplos. En la clasificación de quiebra, la muestra de } \\
\text { entrenamiento se divide recursivamente en un árbol de decisión en el que los } \\
\text { nodos finales contienen empresas de un solo tipo, en bancarrota o sanas. }\end{array}$ \\
\hline $\begin{array}{l}\text { Razonamiento } \\
\text { basado en casos o } \\
\text { case-based } \\
\text { reasoning (CBR) }\end{array}$ & $\begin{array}{l}\text { Resuelve un nuevo problema de clasificación con la ayuda de casos similares } \\
\text { resueltos previamente. }\end{array}$ \\
\hline $\begin{array}{l}\text { Redes neuronales o } \\
\text { neural networks } \\
\text { (NN) }\end{array}$ & $\begin{array}{l}\text { Las redes neuronales realizan tareas de clasificación inspirándose en los procesos } \\
\text { cerebrales. Las neuronas son nodos con interconexiones ponderadas organizados } \\
\text { en capas. Cada nodo en la capa de entrada es un elemento de procesamiento } \\
\text { que recibe una variedad de señales de entrada de objetos de origen, como puede } \\
\text { ser información sobre empresas, en el caso de predicción de la quiebra, y las } \\
\text { convierte en una única señal de salida. }\end{array}$ \\
\hline $\begin{array}{l}\text { Algoritmos } \\
\text { genéticos o genetic } \\
\text { algorithms (GA) }\end{array}$ & $\begin{array}{l}\text { Funcionan como una técnica de búsqueda estocástica para encontrar una } \\
\text { solución óptima a un problema determinado a partir de una gran cantidad de } \\
\text { soluciones. Para resolver un problema de clasificación como la quiebra, los } \\
\text { investigadores extraen un conjunto de reglas o condiciones usando algoritmos } \\
\text { genéticos. Estas condiciones están asociadas con ciertos puntos de corte. Con } \\
\text { base en estas condiciones, el modelo predeciría si una empresa puede o no ir a la } \\
\text { quiebra. }\end{array}$ \\
\hline $\begin{array}{l}\text { Máquinas de } \\
\text { vectores de soporte } \\
\text { o support vector } \\
\text { machine (SVM) }\end{array}$ & $\begin{array}{l}\text { Emplea un modelo lineal para diseñar un hiperplano de separación óptimo } \\
\text { utilizando un mapeo altamente no lineal de vectores de entrada en un espacio de } \\
\text { características de alta dimensión. Las variables más cercanas al hiperplano se } \\
\text { denominan vectores de soporte y se utilizan para definir el resultado binario, } \\
\text { quebrado o solvente, de las empresas evaluadas. }\end{array}$ \\
\hline
\end{tabular}

Fuente: elaboración propia con base en Alaka et al. (2017), Ravi Kumar y Ravi (2007) y Adnan Aziz y Dar (2006).

\section{Tendencias de investigación emergentes}

Todos los análisis realizados anteriormente permiten determinar las tendencias de investigación existentes respecto a la temática de quiebra empresarial. Uno de los tópicos más recurrentes corresponde al “entrepreneurship", es decir, el emprendimiento, que básicamente se refiere al soporte y apoyo del espíritu empresarial en las sociedades, de tal manera que los empresarios tengan la oportunidad de empezar nuevamente, después de una situación de quiebra empresarial. Autores como Lee et al. (2011) han establecido que, entre más empresarios se 
logren recuperar de situaciones de quiebra, mayor será el porcentaje de nuevas empresas en una determinada nación.

Un segundo tema ampliamente abordado en la literatura sobre quiebra empresarial, son los modelos de predicción de quiebra empresarial más actuales, es decir, aquellos que están basados en sistemas expertos e inteligencia artificial como, por ejemplo, los modelos de redes neuronales, los cuales resultaban ser mucho más confiables que los antiguos modelos basados en técnicas estadísticas (Odom y Sharda, 1990). También es importante destacar el tema de los algoritmos genéticos, temática que, según lo planteado por Shin y Lee (2002), aumenta significativamente el número de publicaciones en aplicaciones como la selección de cartera y de inversiones, la predicción de la quiebra, la evaluación y aprobación de préstamos y la asignación de presupuestos.

Finalmente, el último de los temas que cobra un gran protagonismo en cuanto a las publicaciones existentes al respecto se refiere a la técnica de aprendizaje automático denominado "máquinas de vectores de soporte" que, según lo planteado por Min y Lee (2005), consiste en una mezcla de las ventajas de los métodos estadísticos tradicionales con los métodos de aprendizaje robustos basados en datos.

\section{Conclusiones}

A lo largo de este documento fue efectuada una revisión de la literatura científica existente en cuanto a la temática de quiebra empresarial, cubriendo un periodo que va desde el año 1950 hasta el año 2017. El análisis cienciométrico ejecutado incluyó un análisis de coocurrencia de palabras clave y un análisis de redes de citación, con los cuales se obtuvieron los respectivos mapas de conocimiento sobre el tema objeto de estudio. 
La evolución de los documentos publicados evidencia las temáticas referentes a la teoría económica, en los cuales se estudian cuestiones como la estructura de capital y la teoría de la valoración del riesgo de crédito. También se incluyen publicaciones que abordan el desarrollo de modelos para la predicción de la quiebra empresarial, mediante el uso y análisis de datos contables; modelos de predicción haciendo uso del análisis univariante, multivariante y discriminante, y los modelos de predicción más actuales que se soportan en máquinas de vectores de soporte, algoritmos genéticos o redes neuronales.

El análisis permitió observar una clara división en los enfoques de investigación desarrollados; por un lado, los trabajos centrados en las teorías de la estructura de capital, la valoración del crédito y el endeudamiento y, de otra parte, las publicaciones enfocadas en los modelos y técnicas aplicadas en la predicción de la quiebra empresarial. Esta separación resulta muy evidente al observar los diferentes mapas de conocimiento construidos.

Finalmente, cabe destacar que, de los tópicos relacionados con los modelos de predicción de quiebra, resultan muy prometedoras las técnicas de algoritmos genéticos y de máquinas de vectores de soporte, las cuales han sido ampliamente utilizadas en la predicción de quiebra en bancos y en empresas, sobre todo la segunda opción, que mezcla las principales ventajas y fortalezas de los métodos estadísticos clásicos con métodos de aprendizaje automáticos.

\section{Referencias}

Adnan Aziz, M. y Dar, H. A. (2006). Predicting corporate bankruptcy: where we stand? Corporate Governance: The International Journal of Business in Society, 6(1), 1833. https://doi.org/10.1108/14720700610649436 
Alaka, H. A., Oyedele, L.O., Owolabi, H.A., Kumar, V., Ajayi, S.O., Akinade, O.O. y Bilal, M. (2017). Systematic review of bankruptcy prediction models: Towards a framework for tool selection. Expert Systems with Applications, 94, 164-184. https://doi.org/10.1016/j.eswa.2017.10.040

Altman, E. I. (1968). Financial ratios, discriminant analysis and the prediction of corporate bankruptcy. The Journal of Finance, 23(4), 589-609. https://doi.org/10.2307/2978933

Altman, E. I. y McGough, T. P. (1974). Evaluation of a company as a going concern. Journal of Accountancy, 138(6), 50-57.

Altman, E. I. (1984). A further empirical investigation of the bankruptcy cost question. The Journal of Finance, 39(4), 1067-1089. https://doi.org/10.1111/j.1540-6261.1984.tbo3893.x

Altman, E. I. y Hotchkiss, E. (2010). Corporate financial distress and bankruptcy: Predict and avoid bankruptcy, analyze, and invest in distressed debt. John Wiley \& Sons.

Andrade, G. y Kaplan, S. N. (1998). How costly is financial (not economic) distress? Evidence from highly leveraged transactions that became distressed. The Journal of Finance, 53 (5), 1443-1493. https://doi.org/10.1111/0022-1082.00062

Ang, J. S., y Chua, J. H. (1981). Corporate bankruptcy and job losses among top level managers. Financial Management, 10 (5), 70-74. https://doi.org/10.2307/3664858

Balcaen, S. y Ooghe, H. (2006). 35 years of studies on business failure: an overview of the classic statistical methodologies and their related problems. The British Accounting Review, 38(1), 63-93. https://doi.org/10.1016/j.bar.2005.09.001

Beaver, W. H. (1966). Financial ratios as predictors of failure. Journal of Accounting Research, 4, 71-111. https://doi.org/10.2307/2490171

Bharath, S. T. y Shumway, T. (2008). Forecasting default with the Merton distance to default model. The Review of Financial Studies, 21(3), 1339-1369. https://doi.org/10.1093/rfs/hhno44

Black, F. y Scholes, M. (1973). The pricing of options and corporate liabilities. Journal of Political Economy, 81(3), 637-654. 
Bunn, P. y Redwood, V. (2003). Company accounts-based modelling of business failures and the implications for financial stability [documento de trabajo 210]. Bank of England. http://dx.doi.org/10.2139/ssrn.598276

Campbell, J. Y., Hilscher, J. y Szilagyi, J. (2008). In search of distress risk. The Journal of Finance, 63(6), 2899-2939. https://doi.org/10.1111/j.1540-6261.2008.01416.x

Centre for Science and Technology Studies. (2014). CitNetExplorer (versión 1.0.o). Leiden University. https://www.citnetexplorer.nl/

Centre for Science and Technology Studies. (2018). VOSviewer (versión 1.6.9.). Leiden University. https://www.vosviewer.com/

Coats, P. K. y Fant, L. F. (1993). Recognizing financial distress patterns using a neural network tool. Financial Management, 22(3), 142-155.

Crouhy, M., Galai, D. y Mark, R. (2000). A comparative analysis of current credit risk models. Journal of Banking and Finance, 24(1-2), 59-117. https://doi.org/10.1016/S0378-4266(99)00053-9

Dichev, I. D. (1998). Is the risk of bankruptcy a systematic risk? The Journal of Finance, 53(3), 1131-1147. https://doi.org/10.1111/0022-1082.00046

Ferguson, M. F. y Shockley, R. L. (2003). Equilibrium “anomalies”. The Journal of Finance, 58 (6), 2549-2580. https://doi.org/10.1046/j.1540-6261.2003.00615.X

García-Gallego, A. y Mures-Quintana, M. J. (2013). La muestra de empresas en los modelos de predicción del fracaso: influencia en los resultados de clasificación. Revista de Métodos Cuantitativos para la Economía y la Empresa, 15, 133-150. http://hdl.handle.net/10433/372

Gilson, S. C., John, K. y Lang, L. H. (1990). Troubled debt restructurings: An empirical study of private reorganization of firms in default. Journal of Financial Economics, 27(2), 315-353. https://doi.org/10.1016/0304-405X(90)90059-9

Gitman, L. J., Juchau, R. y Flanagan, J. (2015). Principles of managerial finance (6. ${ }^{\mathrm{a}}$ ed.). Pearson Higher Education.

Griffin, J. M. y Lemmon, M. L. (2002). Book-to - market equity, distress risk, and stock returns. The Journal of Finance, 57 (5), 2317-2336. https://doi.org/10.1111/1540$\underline{6261.00497}$ 
Gruber, M. J. y Warner, J. B. (1977). Bankruptcy costs: Some evidence. The Journal of Finance, 32 (2), 337-347. https://doi.org/10.1111/j.1540-6261.1977.tbo3274.x

Hickman, W. B. (1958). Corporate bond quality and investor experience. NBER Books. Princeton University Press.

Hillegeist, S. A., Keating, E. K., Cram, D. P. y Lundstedt, K. G. (2004). Assessing the probability of bankruptcy. Review of Accounting Studies, 9 (1), 5-34. https://doi.org/10.1023/B:RAST.0000013627.90884.b7

Ibarra, A. (2002). Análisis de las dificultades financieras de las empresas en una economía emergente: las bases de datos y las variables independientes en el sector hotelero de la bolsa mexicana de valores [tesis doctoral, Universitat Autònoma de Barcelona). Tesis Doctorals en Xarxa. https://www.tdx.cat/handle/10803/3941\#page=1.

Jensen, M. C. (1986). Agency costs of free cash flow, corporate finance, and takeovers. American Economic Review, 76(2), 323-329. http://dx.doi.org/10.2139/ssrn.99580

Jensen, M. C. y Meckling, W. H. (1976). Theory of the firm: Managerial behavior, agency costs and ownership structure. Journal of Financial Economics, 3,(4), 305-360. DOI: https://doi.org/10.1016/0304-40.5X(76)90026-X

Leland, H. E. (1994). Corporate debt value, bond covenants, and optimal capital structure. The Journal of Finance, 49(4), 1213-1252. https://doi.org/10.1111/j.1540-6261.1994.tbo2452.x

Leland, H. E. y Toft, K. B. (1996). Optimal capital structure, endogenous bankruptcy, and the term structure of credit spreads. The Journal of Finance, 51(3), 987-1019. https://doi.org/10.1142/9789814759595_0003

Lev, B. (1978). Análisis de los Estados Financieros: un nuevo enfoque. Ediciones ESIC.

Lizarraga, F. (1997). Utilidad de la información contable en el proceso de fracaso: Análisis del sector industrial de la mediana empresa española. Revista Española de Financiación y Contabilidad, 26(92), 871-915.

Manzaneque, M., Benegas, R. y García D. (2010). Diferentes procesos de fracaso empresarial: Un análisis dinámico a través de la aplicación de técnicas estadísticas clúster. Revista Europea de Dirección y Economía de la Empresa, 19(3), 67-88.

Revista Activos

ISSN: 0124-5805 | e-ISSN: 2500-5278 | DOI: https://doi.org/10.15332/25005278

Vol. 19 N.o 1 | enero-junio de 2021 
Merton, R. C. (1974). On the pricing of corporate debt: The risk structure of interest rates. The Journal of Finance, 29(2), 449-470. https://doi.org/10.1111/j.15406261.1974.tb03058.x

Min, J. H. y Lee, Y. C. (2005). Bankruptcy prediction using support vector machine with optimal choice of kernel function parameters. Expert Systems with Applications, 28(4), 603-614. https://doi.org/10.1016/j.eswa.2004.12.008

Modigliani, F. y Miller, M. H. (1958). The cost of capital, corporation finance and the theory of investment. The American Economic Review, 48(3), 261-297.

Modigliani, F. y Miller, M. H. (1965). The cost of capital, corporation finance, and the theory of investment: reply. The American Economic Review, 55(3), 524-527.

Myers, S. C. (1977). Determinants of corporate borrowing. Journal of Financial Economics, 5(2), 147-175. https://doi.org/10.1016/0304-405X(77)90015-0

Myers, S. C. y Majluf, N. S. (1984). Corporate financing and investment decisions when firms have information that investors do not have. Journal of Financial Economics, 13(2), 187-221. https://doi.org/10.1016/0304-405X(84)90023-0

Odom, M. D. y Sharda, R. (1990, junio). A neural network model for bankruptcy prediction. En Neural Networks, IJCNN International Joint Conference on (163168). IEEE.

Ohlson, J. A. (1980). Financial ratios and the probabilistic prediction of bankruptcy. Journal of Accounting Research, 18(1), 109-131. https://doi.org/10.2307/2490395

Ooghe, H. y De Prijcker, S. (2008). Failure processes and causes of company bankruptcy: a typology. Management Decision, 46(2), 223-242. https://doi.org/10.1108/00251740810854131

Opler, T. C. y Titman, S. (1994). Financial distress and corporate performance. The Journal of Finance, 49(3), 1015-1040. https://doi.org/10.1111/j.15406261.1994.tbooo86.x

Radhakrishnan, S., Erbis, S., Isaacs, J. A. y Kamarthi, S. (2017). Novel keyword cooccurrence network-based methods to foster systematic reviews of scientific literature. PloS One, 12(9), e0185771. https://doi.org/10.1371/journal.pone.0172778 
Ravi Kumar, P. R. y Ravi, V. (2007). Bankruptcy prediction in banks and firms via statistical and intelligent techniques-A review. European Journal of Operational Research, 180(1), 1-28. https://doi.org/10.1016/j.ejor.2006.08.043

Romero, F. (2013). Alcances y limitaciones de los modelos de capacidad predictiva en el análisis del fracaso empresarial. Revista Ad-Minister, (23), 45-70.

Rose, P. S. y Giroux, G. A. (1984). Predicting corporate bankruptcy: an analytical and empirical evaluation. Review of Business and Economic Research, 19(2), 1-12.

Shleifer, A. y Vishny, R. W. (1992). Liquidation values and debt capacity: A market equilibrium approach. Journal of Finance, 47(4), 1343-1366. https://doi.org/10.1111/j.1540-6261.1992.tbo4661.x

Shiers, A. F. y Williamson, D. P. (1987). Nonbusiness bankruptcies and the law: Some empirical results. Journal of Consumer Affairs, 21(2), 277-292. https://doi.org/10.1111/j.1745-6606.1987.tbo0203.x

Shin, K. S. y Lee, Y. J. (2002). A genetic algorithm application in bankruptcy prediction modeling. Expert Systems with Applications, 23(3), 321-328. https://doi.org/10.1016/S0957-4174(02)00051-9

Shin, K. S., Lee, T. S. y Kim, H. J. (2005). An application of support vector machines in bankruptcy prediction model. Expert Systems with Applications, 28(1), 127-135. https://doi.org/10.1016/i.eswa.2004.08.009

Shumway, T. (2001). Forecasting bankruptcy more accurately: A simple hazard model. The Journal of Business, 74 (1), 101-124. https://doi.org/10.1086/209665

Simpson, P. B. y Anderson, P. S. (1957). Liabilities of business failures as a business indicator. The Review of Economics and Statistics, 39(2), 193-199.

Situm, M. (2014). The age and the size of the firm as relevant predictors for bankruptcy. Journal of Applied Economics and Business, 2(1), 5-30.

Smith, R. F. y Winakor, A. (1935). Changes in the Financial Structure of Unsuccessful Industrial Corporations. University of Illinois.

Smith, R. F., y Winakor, A. H. (1930). A test analysis of unsuccessful industrial companies. Bulletin, 31. 
Tian, S., Yu, Y. y Guo, H. (2015). Variable selection and corporate bankruptcy forecasts. Journal of Banking and Finance, 52, 89-100. https://doi.org/10.2469/dig.v45.n8.13

Van Eck, N. J. y Waltman, L. (2017). Citation-based clustering of publications using CitNetExplorer and VOSviewer. Scientometrics, 111(2), 1053-1070. https://doi.org/10.1007/s11192-017-2300-7

Vassalou, M. y Xing, Y. (2004). Default risk in equity returns. The Journal of Finance, 59 (2), 831-868. https://doi.org/10.1111/j.1540-6261.2004.00650.x

Clarivate. (2018). Web of Science [base de datos]. https://webofscience.udemproxy.elogim.com/wos/woscc/basic-search

Wilson, R. L. y Sharda, R. (1994). Bankruptcy prediction using neural networks. Decision Support Systems, 11(5), 545-557. https://doi.org/10.1016/0167-9236(94)90024-8

Zacharakis A.L., Meyer G. y De Castro, J. (1999). Differing perceptions of new venture failure: A matched exploratory study of venture capitalists and entrepreneurs Journal of Small Business Management, 37(3), 1-14.

Zmijewski, M. E. (1984). Methodological issues related to the estimation of financial distress prediction models. Journal of Accounting Research, 22, 59-82. https://doi.org/10.2307/2490859 